\title{
Photorealistic Rendering of Large Tissue Deformation for Surgical Simulation
}

\author{
Mohamed A. ElHelw, Benny P. Lo, A.J. Chung, Ara Darzi, and \\ Guang-Zhong Yang \\ Royal Society/Wolfson Medical Image Computing Laboratory, \\ Imperial College London, London, United Kingdom \\ \{mohammed.elhelw, benlo, ajchung, a.darzi, \\ g.z.yang\}@imperial.ac.uk
}

\begin{abstract}
With the increasing use of computer based simulation for training and skills assessment, growing effort is being directed towards enhancing the visual realism of the simulation environment. Image-based modelling and rendering is a promising technique in that it attains photorealistic visual feedback while maintaining interactive response. The purpose of this paper is to extend an existing technique for simulating tissues with extensive deformation. We demonstrate that by the incorporation of multiple virtual cameras, geometric proxy and viewing projection manifolds, interactive tissueinstrument interaction can be achieved while providing photorealistic rendering. Detailed steps involved in the algorithm are introduced and quantitative error analysis is provided to assess the accuracy of the technique in terms of projection error through 3D image warping. Results from phantom and reallaparoscope simulation demonstrate the potential clinical value of the technique.
\end{abstract}

\section{Introduction}

In minimal invasive surgery, virtual and augmented realities are increasingly being used as new ways of training, preoperative planning, and navigation. Realistic modelling of soft tissue properties in terms of both mechanical and visual characteristics is essential to the perceptual fidelity of the simulation environment. In practice, this is a challenging task due to the diversity of tissue properties and the lack of non-invasive techniques for mapping mechanical indices in vivo. For surgical simulation, the nature of the problem strides across both computer graphics for photorealistic rendering and biomechanics for tissue property simulation [1]. Whilst major research has been conducted in the measurement and simulation of soft tissue deformation, increasing effort is being directed towards enhancing the visual realism of the simulation environment.

In our previous work, we have presented an image-based rendering approach for simulating soft tissue deformation due to instrument interaction [2]. The method is based on associating a depth map with each colour texture for modelling surface details. Considering the fact that real-time mechanical simulation of tissue- 
deformation is normally restricted to a relatively coarse mesh structure, which cannot provide photo-realistic results if it is rendered directly, macro- and micro-surface structures, i.e. details, are decomposed during a pre-processing step. During simulation, deformation resulting from tissue-instrument interaction is rapidly calculated by modifying the macrostructure. Microstructures are subsequently added by following the 3D image-warping paradigm [3]. It has been shown that the method significantly reduces the polygonal count required to model the scene whilst offering realistic rendering results. Interactive response can be achieved on a normal PC without dedicated hardware.

The purpose of this paper is to extend the modeling and rendering framework for complex scenes with large tissue deformation. Instead of using one virtual camera to sample the scene for 3D image warping, multiple cameras with overlapping field-ofview are used. This ensures rapid photo-realistic rendering during run time whilst maintaining re-projection accuracy even in the presence of large deformation.

\section{Method}

The proposed method is divided into preprocessing and interactive phases. During the first phase, tissue geometry and colour information are obtained and made amenable for interactive processing. In the interactive phase, realistic deformation behaviour is achieved by using mass-spring modeling while image-based rendering is used to attain photorealistic visual feedback. In this section we describe in detail the tasks performed at each phase. We also introduce a number of new concepts that make an interactive image-based solution possible for simulating large tissue areas and involve significant deformation. These concepts include the deformable geometric proxy and the viewing projection manifold.

\subsection{Pre-processing Phase}

The pre-processing step in this study is similar to that of our previous work and involves most of the computationally intensive tasks including the construction of a geometric proxy, i.e. a coarse deformable geometric model describing tissue surface and the extraction of surface color and texture details. The output of this stage is the geometric proxy, and a pair of color and microstructure images for each virtual camera.

\subsubsection{Geometry and Colour Information Acquisition}

Several methods for obtaining tissue or organ geometry and colour can be utilised. We have conducted two experiments to test our technique where different acquisition approaches were used. For the first experiment, an artificial phantom model, mimicking large tissue or organ, was constructed. The phantom was made of silicone rubber and the surface was coated with silicone rubber mixed with acrylic to give it a specular finish that looks similar to soft tissue. The tomographic model of the phantom was scanned with a Siemens So-maton Volume Zoom four-channel multi- 
detector CT scanner with a slice thickness of $3 \mathrm{~mm}$ and in-plane resolution of $1 \mathrm{~mm}$. Consequently, model geometry was acquired from the volumetric set by using an isosurface extraction method, namely the marching cubes. The model was also photographed to acquire its colour image.

Subsequently, the colour image of the phantom was aligned with its $3 \mathrm{D}$ geometry using $2 \mathrm{D} / 3 \mathrm{D}$ registration. An intensity-based pixel-oriented similarity measure employing the normalised cross correlation of the $2 \mathrm{D}$ image with the projection of the 3D geometry was optimised until correct alignment was established. The similarity between the colour image $(\mathrm{CI})$ and the projected image $(\mathrm{PI})$ of the volumetric data is given by [4]:

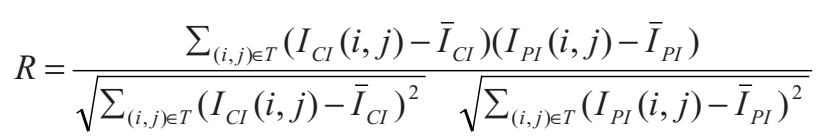

where $\bar{I}_{C I}$ and $\bar{I}_{P I}$ are the mean values of the two images in the overlap region.

For the second experiment, we have used in-vivo data acquired during a laparoscopy procedure. Estimated tissue geometry was attained by using shape-fromshading technique as the surface is assumed to be Lambertian with constant albedo. The depth of the tissue in relation to the camera at each time frame can be estimated with Taylor series expansion, as proposed by Tsai and Shah [5]:

$$
\begin{aligned}
& Z_{t}^{n}(x, y)=Z_{t}^{n-1}(x, y)+\frac{\frac{-f\left(Z_{t}^{n-1}(x, y)\right)}{d f\left(Z_{t}^{n-1}(x, y)\right)}}{d Z(x, y)} \text { where } Z_{0}^{0}(x, y)=0 \\
& \frac{d f}{d Z_{t}^{n}}=\left(\frac{(p+q)\left(p p_{s}+q q_{s}+1\right)}{\sqrt{\left(p^{2}+q^{2}+1\right)^{3}} \sqrt{\left(p_{s}{ }^{2}+q_{s}{ }^{2}+1\right)}}-\frac{p_{s}+q_{s}}{\sqrt{\left(p^{2}+q^{2}+1\right)} \sqrt{\left(p_{s}^{2}+q_{s}^{2}+1\right)}}\right) \\
& p=\frac{\partial Z}{\partial x} \quad q=\frac{\partial Z}{\partial y} \quad p_{s}=\frac{\cos \tau \sin \sigma}{\cos \sigma} \quad q_{S}=\frac{\sin \tau \sin \sigma}{\cos \sigma}
\end{aligned}
$$

where $z_{t}^{n}(x, y)$ represents the depth value of pixel $(x, y)$ at time $t$ after $n$ iteration, and $\tau$ is the tilt of the illuminant and $\sigma$ is the slant of the illuminant.

\subsubsection{Geometry Simplification}

Because of its complexity, mesh simplification has to be applied to the created geometric model to obtain a coarser representation that allows for real-time processing. To get an idea about shape complexity, the geometric proxy obtained from the scanned phantom model originally consisted of 80,744 polygons. It was reduced to a coarse model of only 80 polygons by naïvely sub-sampling the original mesh, as illustrated in Figure 1. Better approximation results can be obtained by using more advanced mesh simplification approaches. However, when deformation 
behavior is not of primary objective, high quality visual rendering can still be achieved while using a very coarse proxy. This is one of the main advantages of using the image-based rendering technique to render the deformed tissue.
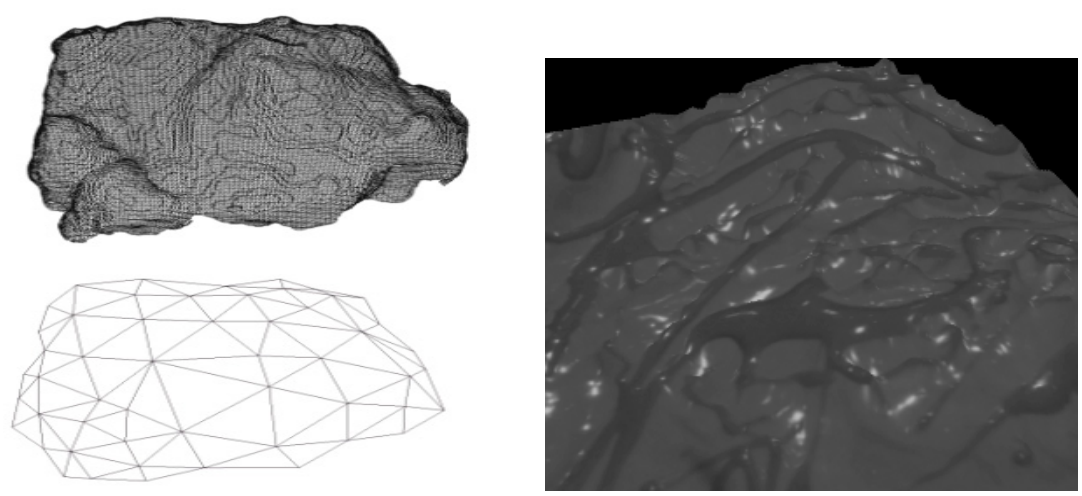

Fig. 1. A mesh reduction technique is applied to the original mesh resulting in a coarser model. Above (left) are the original and coarse meshes. A section of the phantom model rendered by using the image-based technique is shown (right).

After geometry and color information are acquired, the computer-generated model is sampled by a number of virtual cameras with overlapping field-of-view. For each virtual camera, depth and colour information are captured by means of ray casting or by using the graphics hardware depth and color buffers.

\subsubsection{Constructing the Deformable Geometric Proxy}

The deformable geometric proxy is used to rapidly handle deformations resulting from tissue-instrument interactions. It is constructed by fitting masses to the vertices of the coarse geometric model obtained in the previous step, then connecting them by springs and dampers. Generally, approximate deformation models are well suited for surgical training. However, since the proposed image-based solution completely separates deformation modeling from rendering processes, the mass-spring model can be replaced with a finite element model in case accurate deformations are desired.

\subsubsection{Separating Tissue Surface into Macro- and Micro-Structures}

$3 \mathrm{D}$ image warping is used to preserve and enhance the appearance of high-complexity structures on top of tissue surface. As discussed in [2], these details are acquired during the pre-processing phase by using filtering to separate each of the depth or intensity images into macro- and microstructure images then storing the latter. During interactive simulation phase, the macrostructure deformation data is derived from the geometric proxy. Subsequently, microstructures are added to the deformed surface. Thus preserving the microscopic details of the surface undergoing deformation and permitting photorealistic rendering. 


\subsection{Interactive Simulation Phase}

The interactive phase is executed every time step during simulation. It is composed of the five processes described below.

\subsubsection{Handling Interactions and Capturing Deformed Surface Information}

Surgical simulation involves complex interactions such as tissues-instrument, tissuetissue and instrument-instrument interactions [6]. Therefore, the majority of simulation loop cycles are spent on computing collision detection and collision responses. The use of the coarse geometric proxy allows for rapid collision detection necessary for realistic visual and haptic responses by reducing the number of collision calculations. In addition, deforming the geometric proxy results in modifying the macrostructure, i.e. the depth, captured by the virtual cameras sampling the tissue surface. For each virtual camera, the process of reading modified depth can be efficiently carried out by making use of the available graphics hardware. For instance, OpenGL [7] depth buffer can be used to rapidly acquire depth information.

\subsubsection{Adding Microstructure and Rendering Deformed Tissue}

The microstructure information is added to the tissue by first distorting the microstructure image to conform to the deformed macrostructure then modulating the latter with the distorted image. This accounts for depth changes resulting from tiny surface details. The procedure is executed for each virtual camera and the view of the deformed tissue is produced as described in [2]. The generated views are then combined together by using a viewing projection manifold (VPM) which is a triangular surface constructed from the intersection of the virtual cameras viewing planes as illustrated in Figure 2.

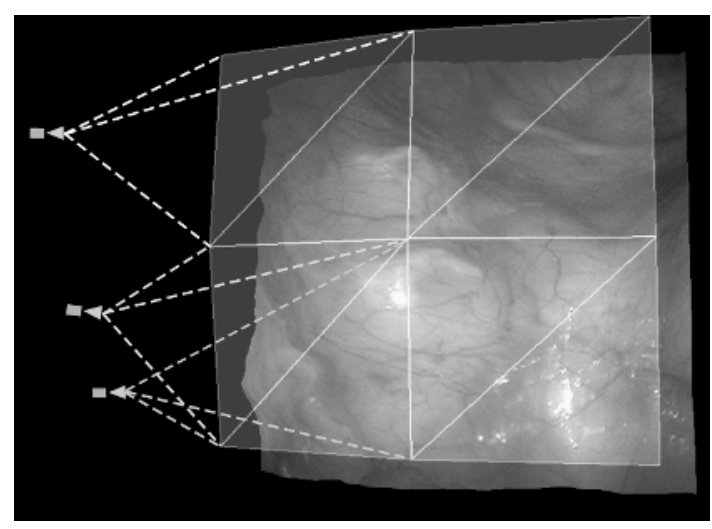

Fig. 2. Viewing projection manifold superimposed over tissue. Also shown several virtual cameras used to capture model depth and colour information 


\section{Results}

\subsection{Visual Feedback}

The image-based technique has been applied to modelling and rendering an artificial phantom model depicting a deformable bulky tissue or organ. Figure 1 (right) illustrates the results obtained where it can be noticed how photorealistic rendering was achieved with reduced modelling efforts.

Figure 3 shows a number of depth and colour images captured by virtual cameras when the technique was applied to in-vivo data. Figure 4 shows a novel view of the tissue. The high fidelity visual feedback using the proposed technique is demonstrated.
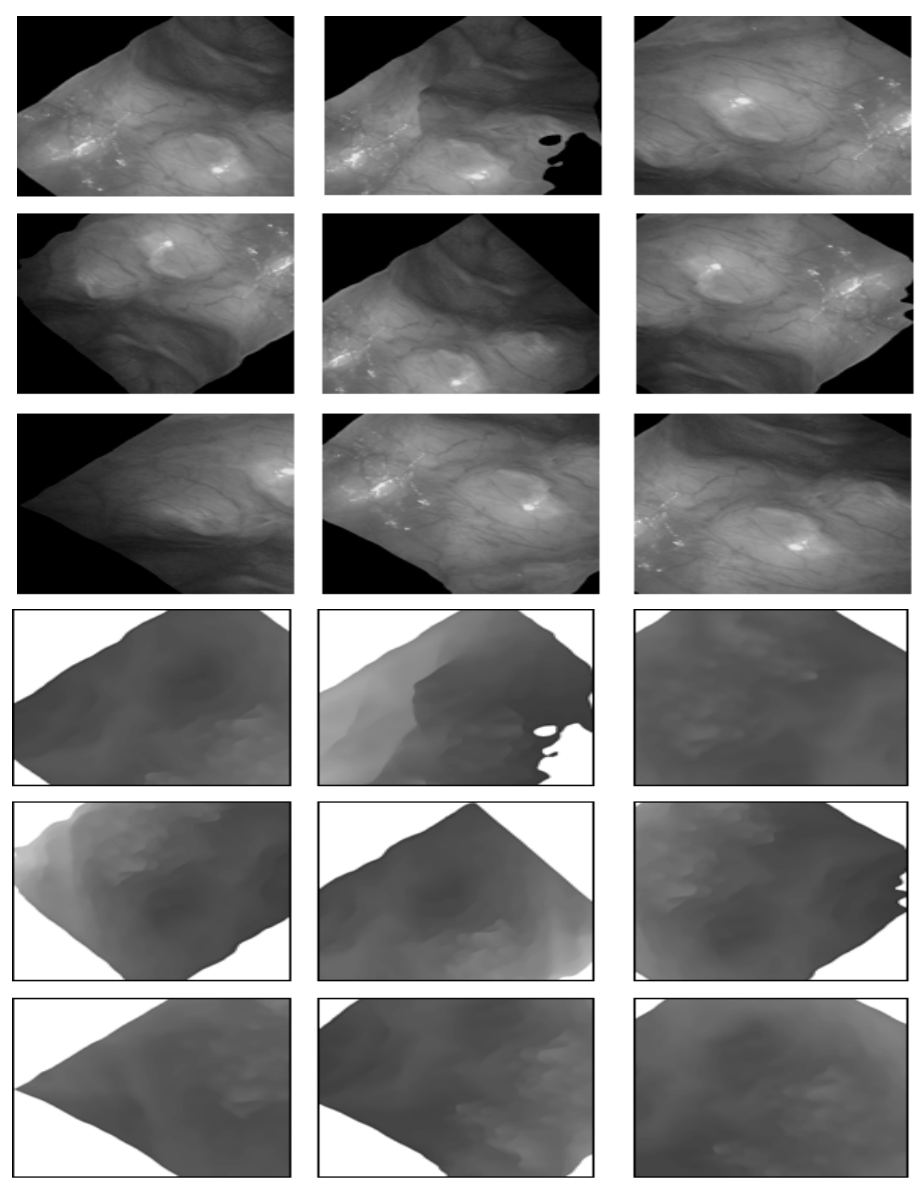

Fig. 3. Colour and depth data captured by virtual cameras. Notice the non-smooth depth resulting from using the shape-from-shading method to extract the geometry of the model [8] 


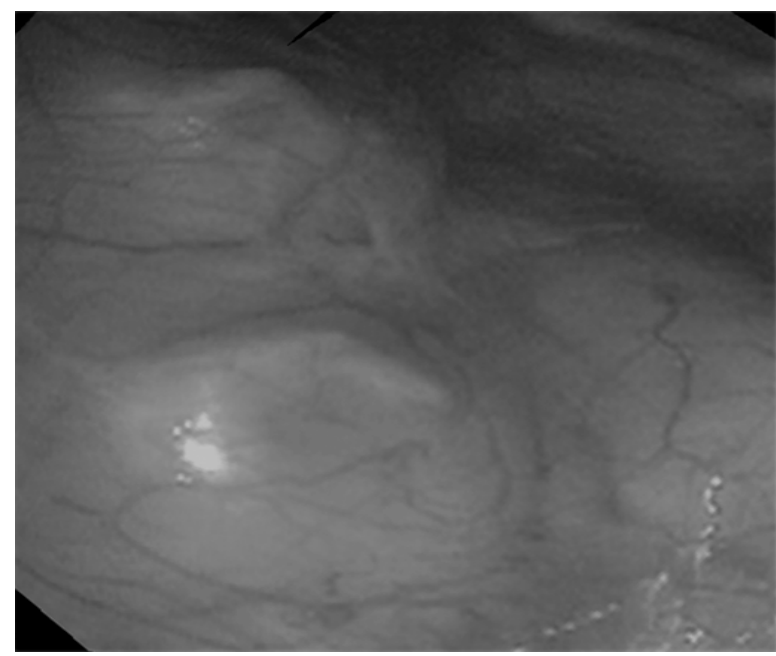

Fig. 4. A novel view of in-vivo tissue rendered with the image-based technique.

\subsection{Validation}

The image-based rendering technique is validated through error analysis by comparing it to the conventional polygonal method. For this study, the "goldstandard" is defined as the screen-space coordinates of the projection of selected original model samples. Thus, error is defined as the deviation, in pixels, of same samples rendered by using the image-based and polygon-based methods. The results of the study are shown in Table 1 where it can be seen that projection errors are minimized when the image-based technique is used. This is expected since the imagebased method uses depth images, which provide more faithful representations of the original model, to render the tissue.

Table 1. Error analysis for comparing the accuarcy of image-based and polygon-based methods at different viewing angles

\begin{tabular}{ccc}
\hline $\begin{array}{c}\text { Viewing Angle } \\
\text { (radians) }\end{array}$ & \multicolumn{2}{c}{ Projection Error in Pixels } \\
\hline 0.705723 & 1.41421 & Polygon Method \\
0.867709 & 1.52315 & 9.14112 \\
1.91832 & 2.34094 & 3.02655 \\
2.44696 & 2.20907 & 16.1307 \\
2.44881 & 1.20 & 10.1193 \\
\hline
\end{tabular}




\section{Discussion and Conclusions}

In this paper we described an image-based rendering method for modelling and rendering bulky tissues and organs. In order to maintain efficient execution, the method is divided into pre-processing and interactive phases. At the pre-processing phase, computationally demanding tasks such as acquiring and constructing the geometric proxy, and separating and storing tissue information are carried out. During simulation, interactivity is sustained by using the coarse geometric proxy to compute tissue instrument interactions, whereas photorealistic rendering is achieved by using image-based rendering to augment 3D surface details and render the final deformed tissue. Although the proposed method provides a photorealistic rendering quality, it assumes fixed tissue topology such that only limited tissue-instrument interaction operations can be simulated. Extending the method to handle other types of interaction such as cutting and piercing is to be investigated. Another area for future research is exploring the use of emerging methods for acquiring in-vivo tissue geometry such as the use of projected structured lighting techniques [9] to extract multiple depth images, which can then be combined to obtain model geometry [10].

\section{References}

1. Delingette, H. Towards Realistic Soft Tissue Modeling in Medical Simulation. INRIA. Report No 3506 (1998). http://www.inria.fr/Equipes/EPIDAURE-eng.html

2. ElHelw, M.A., Chung, A.J., Darzi, A. and Yang, G.Z. Image-Based Modelling of Soft Tissue Deformation. MICCAI03 (2003) 83-90

3. McMillan, L. An Image-Based Approach to Three-Dimensional Computer Graphics. Ph.D. Dissertation. UNC Computer Science Technical Report TR97-013 (1997)

4. Penney, P.G., Weese J., Little, J., Desmedt, P., Hill, D. and Hawkes, D. A Comparison of Similarity Measures for Use in 2D-3D Medical Image Registration. MICCAI98 (1998) 1153-1161

5. Tsai, P.S. and Shah, M. Shape From Shading Using Linear Approximation. Image and Vision Computing Journal, Vol. 12 No. 8 (1994) 487-498

6. Conference Course Notes on Surgical Simulation. Medicine Meets Virtual Reality (2002)

7. Neider J., Davis, T. and Woo, M. OpenGL Programming Guide. 2nd edn. Addison Wesley (1997)

8. Stoyanov, D., ElHelw, M., Lo, B.P., Chung, A., Bello, F. and Yang, G.Z. Current Issues of Photorealistic Rendering for Virtual and Augmented Reality in Minimally Invasive Surgery. Seventh International Conference on Information Visualization (IV'03), London, England (2003) 350-358

9. Keller, K. and Ackerman, J. Real-time Structured Light Depth Extraction. Three Dimensional Image Capture and Applications III, SPIE proceedings (2000) 11-18

10. Huber, F.D. Automatic 3D Modeling Using Range Images Obtained from Unknown viewpoints. Proceedings of the Third International Conference on 3-D Digital Imaging and Modeling, IEEE Computer Society (2001) 153-160 